REVISIÓN

\title{
Uso seguro y riesgos microbiológicos del agua residual para la agricultura
}

\author{
Safe use and microbiological risks of wastewater for \\ agriculture
}

\author{
María Isabel González González ${ }^{\mathrm{I}}$, Sergio Chiroles Rubalcaba ${ }^{\mathrm{II}}$ \\ ILicenciada en Ciencias Biológicas. Doctor en Ciencias de la Salud. Investigador \\ Titular. . Instituto Nacional de Higiene, Epidemiología y Microbiología (INHEM). La \\ Habana, Cuba. \\ IILicenciado en Microbiología. Máster en Microbiología. Investigador Agregado. \\ Instituto Nacional de Higiene, Epidemiología y Microbiología (INHEM). La Habana, \\ Cuba.
}

\section{RESUMEN}

El agua dulce es un recurso vital pero cada día está más escaso debido al crecimiento demográfico, la urbanización y la industrialización, a lo que se suman los conflictos asociados a los cambios climáticos. El propósito de este estudio es aportar información actualizada sobre las guías de la OMS para el uso seguro de las aguas residuales en la agricultura. Se identificaron 33 documentos relacionados con el tema, de ellos se seleccionaron 13 publicados entre 1994 y 2007, que contemplan informes de la UNESCO, guías y manual de la OMS y revisiones críticas del tema. Se resume información sobre las guías de la OMS y la importancia de su incorporación paulatina en Cuba, se destacan, dentro de los Objetivos del Desarrollo del Milenio, aquellos relacionados con el uso seguro de aguas residuales y excretas para la agricultura y acuicultura, que justifican la actual importancia de las nuevas guías propuestas. El uso de aguas residuales en la agricultura puede aportar beneficios, pero su uso no controlado generalmente está relacionado con impactos negativos sobre la salud humana. En Cuba, el reuso del agua es poco utilizado pues si bien la agricultura urbana se ha desarrollado últimamente, se realiza con agua potable. Se debe considerar en el país las guías de la OMS para la futura valoración del uso de las aguas residuales tratadas en la agricultura sin riesgo para la salud y obtener resultados beneficiosos para la población y el ambiente. En resumen, se enumeran aspectos a considerar para el uso seguro de las aguas residuales en la agricultura en Cuba, necesarios para implementar

http://scielo.sld.cu 
programas nacionales de vigilancia sanitaria y de agricultura con énfasis en los aspectos microbiológicos.

Palabras clave: Reuso, agua residual, agricultura, riesgos microbiológicos, guías de la OMS.

\begin{abstract}
Drinking water is a vital but increasingly dwindling resource due to the population growth, housing planning, industrialization associated to new potential demands upon the existing water resources, to which climatic change-related events are added. The objective of this study was to provide updated information on the WHO guidelines for the safe use of wastewater in agriculture. A total of 33 documents about this topic were identified; 13 of them published from 1994 to 2007 were selected since they included UNESCO reports, WHO guidelines and manual and critical reviews. Information was summarized on the WHO guidelines and the importance of their gradual application in Cuba. Among the Millennium Development Goals, those related to the safe use of wastewater and excreta for agriculture and aquaculture were underscored because they supported the present importance of the new suggested guidelines. The use of wastewaters in agriculture may bring about benefits, but their use without restriction is generally related to negative impact on the human health. The water reuse is not very common in Cuba; although the agriculture in urban areas has developed in the last few years, it depends on drinking water. The WHO guidelines must be taken into consideration for the future assessment of the use of treated wastewater in agriculture without causing any risk for health, and for the benefits it may bring to the environment and the population as well. In summary, some aspects to be considered to safely use wastewater in the Cuban agriculture, which are also necessary to implement national health and agricultural surveillance programs centered on microbiological aspects, were listed.
\end{abstract}

Key words: Reuse, wastewater, agriculture, microbiological risks, WHO guidelines.

El mundo está trasformándose:

de estar basado en una población principalmente rural a una predominantemente urbana; eso hace que las ciudades necesiten más y más agua para la agricultura. Sin embargo, la mayor parte del agua que se usa en las áreas urbanas viene de las cloacas mismas

Dr. Colin Chartres Director General del Instituto Internacional de la Gestión del Agua. ${ }^{1}$

\title{
INTRODUCCIÓN
}

http://scielo.sld.cu 
El agua dulce es un recurso vital, pero cada día más escaso. Esta escasez es originada por el crecimiento demográfico, la urbanización y la industrialización asociada a nuevas demandas potenciales sobre los recursos hídricos existentes, a lo que se suman los conflictos asociados a los cambios climáticos, de ahí que el reuso creciente de aguas residuales para la agricultura, la acuicultura, la recarga de aguas subterráneas y otros usos se planifique y se incremente paulatinamente.

Según la UNESCO ${ }^{2}$ cada día los ecosistemas acuáticos están siendo dañados de forma irreparable debido al crecimiento de la población, al desarrollo de infraestructuras, a la conversión del uso de la tierra, la sobreexplotación agrícola, la introducción de especies exóticas y la contaminación. Cada día, dos millones de toneladas de desechos se vierten en los canales. Desde el año 1900 ha desaparecido el $50 \%$ de los humedales del mundo, así como una gran cantidad de especies de peces, pájaros y mamíferos.

Se plantea que a medida que crece la población mundial, la presión ejercida sobre los recursos hídricos es mayor. Durante el siglo pasado, la población mundial se triplicó, mientras que el consumo de agua se sextuplicó. Además, se prevé para el año 2050, que una de cada cuatro personas viva en un país afectado por la escasez de agua dulce crónica o recurrente, siendo la situación más alarmante en el África Subsahariana: se estima que en el año 2025 casi 230 millones de africanos se enfrentarán a la escasez de agua y 460 millones vivirán en países con escasez de agua.

Además, si se considera que cada litro de agua residual contamina al menos 8 litros de agua dulce, se estima que, anualmente unos $12000 \mathrm{~km}^{3}$ de recursos hídricos del planeta no están disponibles para su aprovechamiento. Si esta cifra avanza al mismo ritmo que el crecimiento de la población, que se estima alcanzará los 9000 millones de personas para 2050, el planeta perdería cada año unos $18000 \mathrm{~km}^{3} \mathrm{de}$ recursos hídricos. En la actualidad, solo el $10 \%$ de las aguas residuales domésticas son recolectadas en los países en vías de desarrollo y sólo el $10 \%$ de las plantas de tratamiento de aguas residuales existentes operan de manera fiable y eficiente. ${ }^{3}$

Dentro de los Objetivos del Desarrollo del Milenio existen dos de ellos relevantes que se relacionan con el uso seguro de aguas residuales y excretas para la agricultura y acuicultura y justifican la actual importancia de las nuevas guías propuestas. Ellos son: ${ }^{4}$

Objetivo 1: Eliminar la extrema pobreza y el hambre. Ej. con el riego de aguas residuales se obtendrían:

- Mayor cantidad de alimentos cosechados para la población.

- Más provecho para los agricultores.

Objetivo 7: Asegurar la sostenibilidad ambiental. Ej. con el riego de aguas residuales se obtendrían:

- Protección de las aguas subterráneas.

- El contenido de nutrientes beneficia el incremento de la cosecha y reduce la cantidad de agua residual no tratada que se descarga en el ambiente acuático.

¿Por qué la necesidad del reuso de aguas residuales? 
1. Cada día el agua es más escasa para sus diferentes usos, principalmente en países de climas secos o en regiones que padecen eventos de extrema sequía.

2. Por la escasez del agua, existen conflictos políticos y sociales.

3. El aprovechamiento de estas aguas para la agricultura y acuicultura de forma segura, garantizan una mayor cobertura de alimentos para la población, cada vez mayor, sobre todo en países en vías de desarrollo.

4. La agricultura es una de las actividades humanas que consumen más agua, si se usan las aguas residuales de forma segura, esto permitiría una racionalización de las aguas dulces para otros usos, en especial para el consumo humano.

5. Por otra parte, el reuso de esta agua de forma segura, mejoraría los ecosistemas acuáticos y por ende, la situación higiénica ambiental de muchos países.

En algunos casos, las aguas residuales son el único recurso hídrico de las comunidades pobres que subsisten por medio de la agricultura tradicional. Si bien el uso de aguas residuales en la agricultura puede aportar beneficios (incluidos los beneficios de salud como una mejor nutrición y provisión de alimentos para muchas personas), su uso no controlado generalmente está relacionado con impactos negativos importantes sobre la salud humana. Estos impactos en la salud se pueden minimizar cuando se implementan buenas prácticas de manejo en el reuso.

En Cuba, el reuso es poco utilizado, pero al igual que en otros países de la Región, es de importancia notable tratar de incorporar estos conocimientos e implementar sistemas de riego con el uso de agua residual tratada sin riesgo para la salud, y por ende, obtener los resultados beneficiosos para la población y el ambiente.

El objetivo de este trabajo es aportar información actualizada sobre las guías de la OMS, para el uso seguro de las aguas residuales en la agricultura con vistas a su posible adecuación e implantación en los lineamientos de la vigilancia sanitaria con énfasis en los aspectos microbiológicos. Se identificaron un total de 33 documentos relacionados con el tema, de ellos se seleccionaron 13 publicados entre 1994 y 2007, que contemplan informes de la UNESCO, guías y manual de la OMS y revisiones críticas del tema.

\section{SÍNTESIS DE LOS DATOS}

\section{Guías de la (OMS) de las aguas residuales y excretas para la agricultura y acuicultura}

Los antecedentes de las Guías de la OMS son:

- 1918. Guías establecidas por California.

- 1973. Primera versión de la OMS denominada "Reuso de efluentes: métodos de tratamiento de residual y seguridad para la Salud Pública. Reporte de una reunión de expertos de la OMS."

- 1989. Segunda versión, basada en las guías de Engelberg y denominadas "Guías de uso seguro de aguas residuales y excretas en la agricultura y acuicultura.

Reporte de un Grupo Científico de la OMS."

http://scielo.sld.cu 
- 2005-2006. Última versión. Guías para el uso seguro de aguas residuales, excreta y aguas grises, integrada por los siguientes volúmenes:

Volumen I. Aspectos de Política y Regulación.

Volumen II. Uso de aguas residuales en agricultura.

Volumen III. Uso de aguas residuales y excreta en acuicultu

Volumen IV. Uso de excretas y aguas grises en agricultura.

Volumen V. Aspectos de muestreo y de laboratorio (aún en elaboración).

El objetivo fundamental de estas guías es la protección a la salud pública con la prevención de la transmisión por vía hídrica de la enfermedad y son la base para la elaboración de las normativas en cada país, de ahí que tengan dos componentes fundamentales: ${ }^{4}$

1. El componente de salud, que define el nivel de protección a la salud expresado en las metas de salud según el riesgo.

2. El componente de implementación que establece el monitoreo y el aseguramiento del sistema, las responsabilidades institucionales y de supervisión, la documentación y la confirmación del cumplimiento por medio de una vigilancia independiente.

Las guías para el uso seguro de aguas residuales en la agricultura deben encontrar el balance justo entre la maximización de los beneficios de salud pública y las ventajas de usar recursos escasos. Es necesario que las Guías sean lo suficientemente flexibles para poder adaptarlas a las condiciones locales, sociales, económicas y ambientales de los países. Además, se deben implementar paralelamente con otras intervenciones de salud como la promoción de la higiene, los servicios de agua potable y saneamiento adecuados y otras medidas de atención primaria de la salud.

El uso de aguas residuales para el riego de cultivos es cada vez más común. El rendimiento de los cultivos es superior, ya que las aguas residuales contienen nutrientes para el desarrollo de las plantas. Sin embargo, existe el riesgo de que el riego con aguas residuales facilite la transmisión de enfermedades relacionadas con nemátodos intestinales y bacterias fecales a consumidores y agricultores.

Las vías de transmisión o de exposición a patógenos o contaminantes con el uso de aguas residuales para la agricultura y acuicultura son: ${ }^{4,5}$

1. Por contacto con el residual (o cosechas contaminadas) antes, durante o después del riego (trabajadores agrícolas, sus familiares, vendedores y comunidades vecinas).

2. Por inhalación de aerosoles de aguas residuales (trabajadores, comunidades vecinas).

3. Consumo de productos contaminados regados con agua residual.

http://scielo.sld.cu 
4. Consumo de agua de bebida contaminada como resultado de actividades relacionadas con agua residual (acuíferos o aguas superficiales con infiltración de contaminantes químicos o microorganismos patógenos).

5. Consumo de animales (por ej. carne de res o de cerdo) o productos de animales (por ej. leche) que han sido contaminados a través de su exposición con el residual.

6. Por vectores presentes como resultado del desarrollo y manejo de lo esquemas de riego de residual y lagunas de estabilización.

Algunos ejemplos de microorganismos presentes en aguas residuales y la enfermedad que causan se muestran en la tabla $1 .{ }^{5,6}$

Tabla 1. Principales organismos patógenos que pueden estar presentes en aguas residuales

\begin{tabular}{|c|c|}
\hline Organismo patógeno & Enfermedad \\
\hline \multicolumn{2}{|l|}{ Helmintos } \\
\hline $\begin{array}{l}\text { Ascaris hmbricoides, Ancyloston, } \\
\text { duodenale } \\
\text { Ancylostoma spp. } \\
\text { Necator americanus } \\
\text { Strongyioides stercoralis } \\
\text { Trichuris trichiura } \\
\text { Taenia spp } \\
\text { Enterobius vermicularis } \\
\text { Echinococcus granulosus } \\
\text { Schistosoma spp. } \\
\text { Fasciola heoatica, F.gigantica }\end{array}$ & $\begin{array}{l}\text { Ascariasis } \\
\text { Anquilostosomiasis } \\
\text { Larva migrante cutánea } \\
\text { Necatoriasis } \\
\text { Estrongiloidiasis } \\
\text { Trichuriasis } \\
\text { Teniaisis } \\
\text { Enterobiasis } \\
\text { Hidatidosis } \\
\text { Schistosomiasis } \\
\text { Facioliasis }\end{array}$ \\
\hline \multicolumn{2}{|l|}{ Protozoos } \\
\hline $\begin{array}{l}\text { Giardia lamblia } \\
\text { Entamoeba hystolitica } \\
\text { Balantidium coli } \\
\text { Crytosporidium parvum } \\
\text { Cyclospora cayetanensis } \\
\text { Microsporidia }\end{array}$ & $\begin{array}{l}\text { Giardiasis } \\
\text { Disentería amebiana } \\
\text { Balantidiosis } \\
\text { Criptosporidiasis } \\
\text { Trastomos intestinales } \\
\text { Diarrea }\end{array}$ \\
\hline \multicolumn{2}{|l|}{ Bacterias } \\
\hline $\begin{array}{l}\text { Salmonella Tyohi } \\
\text { Salmonella spp. } \\
\text { Shigella spo. } \\
\text { Campyiobacter jejuni } \\
\text { Helicobacter pylori } \\
\text { Escherichia coli } \\
\text { Vibrio cholerae } \\
\text { Legionella pneumophila } \\
\text { Yersinia enterocolitica } \\
\text { Leptospira spp. }\end{array}$ & $\begin{array}{l}\text { Fiebre tifoidea } \\
\text { Salmonelosis } \\
\text { Shigellosis } \\
\text { Gastroenteritis } \\
\text { Gastroenteritis, úloera gástrica } \\
\text { Gastroenteritis } \\
\text { Cólera } \\
\text { Legionelosis/Fiebre de Pontiac } \\
\text { Yersiniosis } \\
\text { Leptospirosis }\end{array}$ \\
\hline \multicolumn{2}{|l|}{ Virus } \\
\hline $\begin{array}{l}\text { Virus de la hepatitis A y E } \\
\text { Rotavirus } \\
\text { Enterovirus } \\
\text { Parvovirus } \\
\text { Adenovirus }\end{array}$ & $\begin{array}{l}\text { Hepatitis infecciosa } \\
\text { Gastroenteritis } \\
\text { Gastroenteritis, meningitis } \\
\text { Gastroenteritis } \\
\text { Infecciones respiratorias, } \\
\text { gastroenteritis }\end{array}$ \\
\hline
\end{tabular}

Esta información es muy útil para relacionar las enfermedades existentes en el país y la región y establecer posibles estudios en las zonas de riego con agua residual tratada. Tres tipos de evaluaciones son empleadas para conocer el riesgo para la salud: los análisis químicos y microbiológicos, fundamentalmente en aguas que presenten considerables efluentes industriales y en el análisis de los lodos procedentes del aprovechamiento de las aguas residuales, que son apropiados si se tienen en cuenta su evaluación sanitaria y las medidas de protección adecuadas para evitar el riesgo a la salud; los estudios epidemiológicos (que en la actualidad son bien escasos) y la evaluación cuantitativa del riesgo, con mayor énfasis en los aspectos microbiológicos, e decir, si el objetivo fundamental de la técnica de

http://scielo.sld.cu 
tratamiento de las aguas residuales es su aprovechamiento en la agricultura o acuicultura se debe evaluar la remoción de patógenos tales como Vibrio cholerae o brotes de determinada enfermedad.

Un resumen de la información disponible basado en estudios epidemiológicos de la transmisión de enfermedades por el uso de aguas residuales en la agricultura se muestra en la tabla $2 .^{5}$

Tabla 2. Resumen de riesgos para la salud asociados con el uso de agua residual para riego

\begin{tabular}{|c|c|c|c|}
\hline Grupo expuesto & Infecciones por helmintos & $\begin{array}{c}\text { Infecciones } \\
\text { por bacterias y virus }\end{array}$ & $\begin{array}{l}\text { Infecciones } \\
\text { por protozoos }\end{array}$ \\
\hline Consumidores & Significativo & $\begin{array}{l}\text { Cólera, fiebre tifoidea, } \\
\text { shigellosis se han } \\
\text { reportado. Incremento } \\
\text { de riesgo si la calidad del } \\
\text { agua es } \geq 10^{4} \mathrm{CT} / 100 \mathrm{~mL} \text {. }\end{array}$ & $\begin{array}{l}\text { Evidencia en superficies } \\
\text { de vegetales pero no hay } \\
\text { evidencia directa de la } \\
\text { transmisión de la enfermedad. }\end{array}$ \\
\hline $\begin{array}{l}\text { Trabajadores } \\
\text { agrícolas } \\
\text { y sus familiares }\end{array}$ & Significativo & $\begin{array}{l}\text { Incremento de riesgo a } \\
\text { enfermedades diarreicas } \\
\text { si la calidad del agua } \\
\text { es } \geq 10^{4} \mathrm{CT} / 100 \mathrm{~mL} \\
\text { Riesgo elevado a infección } \\
\text { por Saimonella en niños } \\
\text { expuestos a agua residual } \\
\text { sin tratar. }\end{array}$ & $\begin{array}{l}\text { Riesgo insignificante } \\
\text { por Giardia intestinalis } \\
\text { por contacto con agua } \\
\text { residual. } \\
\text { Incremento de riesgo } \\
\text { por amebiasis por contacto } \\
\text { con agua residual sin tratar. }\end{array}$ \\
\hline $\begin{array}{l}\text { Comunidades } \\
\text { vecinas }\end{array}$ & $\begin{array}{l}\text { No hay estudios de } \\
\text { riego por aspersión. } \\
\text { Significativo para riego } \\
\text { por flujo o por surco. }\end{array}$ & $\begin{array}{l}\text { Incremento de riesgo. } \\
\text { Un riego por aspersión } \\
\text { con pobre calidad del } \\
\text { agua ( } 10^{6}-10^{3} \mathrm{CT} / 100 \mathrm{~mL} \text { ) } \\
\text { y alta exposición a } \\
\text { aerosoles. }\end{array}$ & No existen evidencias. \\
\hline
\end{tabular}

CT: coliformes termotolerantes (conocidos anteriormente por coliformes fecales).

En algunos países se realiza el riego con agua residual parcialmente tratada o sin tratamiento, lo cual constituye un peligro potencial para la salud humana. Algunos estudios realizados en México reportan la incidencia de enfermedades como ascariasis en grupos con alta exposición ocupacional al riego con agua "cruda". ${ }^{7}$ En una excelente recopilación de estudios realizados en México, se obtienen resultados valiosos para la valoración de los riesgos de los trabajadores agrícolas y las comunidades, donde se relacionan las enfermedades causadas por helmintos con los riesgos del riego con agua residual. ${ }^{8,9}$

En otras regiones, se han realizado investigaciones similares donde se demuestra el impacto para la salud del riego con agua residual. Un estudio interesante es el efectuado en los suburbios de la ciudad de Asmara, Eritrea, África, donde se detectó una alta contaminación de los vegetales con coliformes fecales y quistes de Giardia, así como bacterias patógenas tales como Salmonella spp. y Shigella spp. relacionados con la alta incidencia de gastroenteritis en los trabajadores agrícolas que realizaban el riego con agua residual y consumían dichos vegetales, especialmente repollos y lechugas. ${ }^{10}$ Una investigación más reciente realizada en Dinapur, ${ }^{11}$ ciudad tropical de la India, demostraron la elevada contaminación microbiológica que tienen los vegetales, debido al riego con agua residual

http://scielo.sld.cu 
parcialmente tratada y el riesgo potencial que derivaba esto para la salud de los consumidores.

\section{Metas de salud ${ }^{4,5}$}

Los límites que se han valorado para las guías actuales están basados en estudios epidemiológicos efectuados los cuales no han sido generalizados en diferentes países y se basan en la evaluación del riesgo con respecto a la carga causada por una determinada enfermedad y las metas de salud, calculándose el riesgo tolerable. Este riesgo tolerable está basado en las condiciones locales con el nivel de la salud pública, las prioridades (tipo de enfermedades e importancia relativa) y las capacidades institucionales, económicas, sociales en cada país.

Las metas de salud se definen a partir de una medida estándar de la enfermedad seleccionada (por ej. Rotavirus, Campylobacter jejuni, Crytosporidium parvum) con los Años de Vida Ajustado por Discapacidad (AVAD) conocido en inglés como Disability Adjusted Life Year (DALY) y define el nivel de protección para la salud según el peligro de esa enfermedad siendo su cálculo como:

Riesgo tolerable = AVAD PPPa/AVAD poe

donde:

pppa: por persona por año y pce: por caso de enfermedad

A partir de estos estudios realizados, se ajustó un AVAD de $10^{-6}$ que es la carga estimada de enfermedad asociada con una diarrea leve (por ej. con una tasa de mortalidad de $1 \times 10^{-5}$ ), a un riesgo anual de enfermedad de 1 en 1000 , (aproximadamente 1 en 10 de riesgo a lo largo de la vida), que para una persona sería igual a perder 31,5 segundos en un año, pero para una comunidad significaría perder 1 año por millón de personas.

Este cálculo demostró que el riesgo por infección asociado con rotavirus era mayor que para Campylobacter o Cryptosporidium, de ahí que se indique la reducción de patógenos necesaria para obtener ese AVAD (tabla 3 ). ${ }^{5,6}$ 
Tabla 3. Metas de salud para el uso de agua tratada en la agricultura

\begin{tabular}{|c|c|c|c|}
\hline Escenario de exposición & $\begin{array}{c}\text { Meta de salud } \\
\text { AVAD pppa }\end{array}$ & $\begin{array}{c}\text { Reducción } \\
\text { de patógenos } \\
\text { necesaria }\left(\log _{10}\right)\end{array}$ & $\begin{array}{l}\text { Número de huevos } \\
\text { de helmintos por litro }\end{array}$ \\
\hline Riego sin restricción: & $\leq 10^{-6 a}$ & & \\
\hline Lechuga & & 6 & $\leq 1^{b, c}$ \\
\hline Cebolla & & 7 & $\leq 1^{b, c}$ \\
\hline Riego restringido: & $\leq 10^{-6 a}$ & & \\
\hline Altamente mecanizada & & 3 & $\leq 1^{b, c}$ \\
\hline Mano de obra intensiva & & 4 & $\leq 1^{b, c}$ \\
\hline Riego por goteo: & $\leq 10^{-6 a}$ & & \\
\hline Cultivos de tallo largo & & 2 & Sin recomendación \\
\hline Cultivos de tallo corto & & 4 & $\leq 1^{c}$ \\
\hline
\end{tabular}

a Reducción de rotavirus, ${ }^{b}$ cuando haya niños menores de 15 años expuestos, deben ser tomadas medidas de protección adicionales, por ej. $\leq 0,1$ huevo de helminto, " una media aritmética debe ser determinada durante la estación de riego. El valor de la media $\leq 1$ huevo de helminto debe ser obtenido de al menos el $90 \%$ de las muestras.

Entre las medidas de control consideradas para reducir o evitar el riesgo a la salud se consideran: el tratamiento de las aguas residuales, la restricción de cultivos, el método de riego, la práctica de higiene en el mercado, la preparación de alimentos (incluye el lavado, desinfección, cocción y otros procedimientos), con el consecuente apoyo local o nacional para acometer las diferentes actividades coordinadas por las organismos involucrados. Por ejemplo, en la tabla 4 se observan las diferentes medidas de control y el rango de remoción de patógenos y en la tabla 5 se recomienda la verificación del tratamiento para el monitoreo del agua residual tratada. ${ }^{5,6}$ 
Tabla 4. Reducción de patógenos según las medidas de protección a la salud

\begin{tabular}{|c|c|c|}
\hline Medida de control & $\begin{array}{l}\text { Reducción } \\
\text { Patógenos } \\
\text { (unidades log) }\end{array}$ & Observaciones \\
\hline $\begin{array}{l}\text { Tratamiento de aguas } \\
\text { residuales }\end{array}$ & $1-6$ & $\begin{array}{l}\text { La remoción de patógenos requerida en una plarta de } \\
\text { trataniento de aguas residuales depende de la combinación de } \\
\text { las medidas de protección a la salud. }\end{array}$ \\
\hline $\begin{array}{l}\text { Riego localizado por goteo } \\
\text { (cultivos de tallo bajo) }\end{array}$ & 2 & $\begin{array}{l}\text { Cultivos de raíces en el suelo y cultivos como la lechuga que } \\
\text { crecen muy cercanos al suelo. }\end{array}$ \\
\hline $\begin{array}{l}\text { Riego localizado por goteo } \\
\text { (cultivos de tallo alto) }\end{array}$ & 4 & $\begin{array}{l}\text { Cultivos, tales como el tomate, las partes cosedhadas no están } \\
\text { en contacto con el suelo. }\end{array}$ \\
\hline $\begin{array}{l}\text { Control de emisiones } \\
\text { de aerosoles (riego } \\
\text { por aspersión) }\end{array}$ & 1 & $\begin{array}{l}\text { Uso de micro aspersores, aspersores ontrolados por anemó- } \\
\text { metros, entre otros. }\end{array}$ \\
\hline $\begin{array}{l}\text { Zona buffer de los } \\
\text { aerosoles de los } \\
\text { aspersores (riego } \\
\text { por aspersión) }\end{array}$ & 1 & $\begin{array}{l}\text { Protección de residentes cerca del área de influencia del } \\
\text { aerosol emanado por la aspersión. La zona de amorti- } \\
\text { guamiento debe ser de } 50 \text { a } 100 \mathrm{~m} \text {. }\end{array}$ \\
\hline Muerte de patógenos & 0,5-2 por día & $\begin{array}{l}\text { Muerte natural en la superficie del cultivo que ocurre entre el } \\
\text { útimo riego y el consumo. } \\
\text { La reducción logaritmica depende del clima (temperatura, } \\
\text { intensidad solar, tipo de cultivo y otros. }\end{array}$ \\
\hline Lavado del producto & 1 & Lavado de los cultivos, vegetales y frutas oon agua limpia. \\
\hline Desinfección de producto & 2 & $\begin{array}{l}\text { Lavado con un desinfectante ligero y agua limpia de cultivos } \\
\text { para ensaladas, vegetales y frutas. }\end{array}$ \\
\hline Remoción de cáscara & 2 & Cultivos de frutas o de raices. \\
\hline Cocción del producto & $6-7$ & $\begin{array}{l}\text { Inmersión del cultivo en agua hirviendo hasta que esté } \\
\text { cocinado. }\end{array}$ \\
\hline
\end{tabular}


Tabla 5. Verificación del monitoreo del agua residual según los diferentes niveles de tratamiento y metas de salud para huevos de helmintos

\begin{tabular}{|c|c|c|c|c|c|}
\hline Tipo de riego & Opción & $\begin{array}{l}\text { Reducción } \\
\text { de patógenos } \\
\text { requeridos según } \\
\text { el tratamiento } \\
\text { (unidades log) }\end{array}$ & $\begin{array}{c}\text { Número } \\
\text { de huevos } \\
\text { de helmintos } \\
\text { por litro } \\
\text { (media aritmética) }\end{array}$ & $\begin{array}{l}\text { Escherichis } \\
\text { coli/100 } \mathrm{mL}\end{array}$ & Notas \\
\hline \multirow[t]{3}{*}{ Sin restrioción } & A & 4 & $\leq 1^{b, c}$ & $\leq 10^{3}$ & Cultivo de raíces \\
\hline & $\mathrm{B}$ & 3 & $\leq 1^{b, c}$ & $\leq 10^{4}$ & Cultivo de hojas \\
\hline & C & 2 & $\begin{array}{c}\text { No hay } \\
\text { Recomendación }\end{array}$ & $\leq 10^{5}$ & $\begin{array}{l}\text { Riego por goteo } \\
\text { de cosechas } \\
\text { de tallo largo }\end{array}$ \\
\hline \multirow[t]{5}{*}{ Restringido } & $\mathrm{D}$ & 4 & $\leq 1^{b}$ & $\leq 10^{3}$ & $\begin{array}{l}\text { Riego por goteo } \\
\text { de cosechas } \\
\text { de tallo corto }\end{array}$ \\
\hline & $E$ & 607 & & $\leq 10^{1} 0 \leq 10^{\circ}$ & $\begin{array}{l}\text { El nivel } \\
\text { de verificación } \\
\text { depende de } \\
\text { requerimientos } \\
\text { nacionales o } \\
\text { locales }\end{array}$ \\
\hline & $\mathrm{F}$ & 4 & & $\leq 10^{4}$ & $\begin{array}{l}\text { Agricultura } \\
\text { de labor intensiva }\end{array}$ \\
\hline & $\mathrm{G}$ & 3 & & $\leq 10^{5}$ & $\begin{array}{l}\text { Agricultura } \\
\text { altamente } \\
\text { mecanizada }\end{array}$ \\
\hline & $\mathrm{H}$ & 0,5 & & $\leq 10^{6}$ & $\begin{array}{l}\text { Remodión } \\
\text { de patógenos en } \\
\text { un tanque séption }\end{array}$ \\
\hline
\end{tabular}

a Por ej. Para un tratamiento secundario, filtración y desinfección: $\mathrm{DBO}_{5}<10 \mathrm{mg} / \mathrm{L}$, turbiedad $<2 \mathrm{NTU}, \mathrm{Cl}_{2}$ residual, $1 \mathrm{mg} / \mathrm{L} ; \mathrm{pH}, 6-9 ;$ y coliformes fecales, no detectables en $100 \mathrm{~mL}$, buando haya niñ̃os menores de 15 años expuestos, deben ser tomadas medidas de protección adicionales, por ej. $\leq 0,1$ huevo de helminto, una media aritmética debe ser determinada durante la estación de riego. El valor de la media de $\leq 1$ huevo de helminto debe ser obtenido de al menos el $90 \%$ de las muestras.

\section{Importancia de su incorporación paulatina en Cuba}

La agricultura urbana se ha desarrollado últimamente en Cuba, aunque se realiza con agua potable, se deben considerar estas guías para la futura valoración del uso seguro de las aguas residuales tratadas en la agricultura. Por otra parte, es importante tener en cuenta su empleo en otras áreas, como el riego de césped de parques y campos deportivos, y otros sitios que estén relacionados con su alcance.

En este trabajo se han resumido los aspectos más importantes de estas guías con énfasis en los aspectos microbiológicos. Los métodos de ensayo microbiológicos recomendados: ${ }^{12,13}$

1. Determinación coliformes termotolerantes o Escherichia coli por la técnica de fermentación de tubos múltiples (Número Más Probable/100 mL).

2. Determinación de huevos viables de helmintos (por sedimentación y conteo).

3. Determinación de patógenos bacterianos (en ocasiones, por ejemplo un brote de cólera) por métodos de concentración como el hisopo de Moore.

http://scielo.sld.cu 
Estos métodos son factibles de realizar en los laboratorios de Microbiología de Aguas del país con los recursos necesarios para ellos y no son de alto costo, sin embargo, de un notable beneficio para aportar la información existente en el uso seguro de las aguas residuales tratadas para la agricultura en Cuba. Los valores recomendados no son límites obligatorios pero sí son diseñados para ser empleados en el desarrollo de las estrategias de manejo de riesgo, las cuales pueden incluir normas nacionales o regionales en el contexto ambiental, social, económico y cultural a nivel local o regional.

En general, las medidas adecuadas de protección sanitarias necesitan de la participación de varios organismos y entidades gubernamentales que permitan el trabajo en conjunto con el fin único del uso eficaz del agua en la agricultura y acuicultura y cada país debe tener un programa de vigilancia adecuado y factible de ejecutar, que permita evaluar los sistemas empleados y establecer normativas en relación con el uso de estas aguas y garantizar la protección a la salud y al medio ambiente.

En conclusión, las aguas residuales son un recurso valioso que debe utilizarse sin limitaciones siempre y cuando se tomen las medidas necesarias para proteger la salud y el ambiente. Es importante realizar los estudios químicos y microbiológicos, epidemiológicos y la evaluación cuantitativa de riesgo para garantizar la calidad del agua residual y su uso seguro. En Cuba se ha desarrollado la agricultura urbana pero se realiza fundamentalmente con agua potable, de ahí la importancia de considerar las guías que se ofrecen para la futura valoración del uso de las aguas residuales tratadas en la agricultura y en otras áreas. Es necesario, además, trabajar en el proyecto de un programa de vigilancia factible de ejecutar, que permita establecer las normativas en relación con el uso de estas aguas.

\section{AGRADECIMIENTOS}

A Dania Silva Hernández, trabajadora de la Editorial Ciencias Médicas, por su valiosa ayuda.

\section{REFERENCIAS BIBLIOGRÁFICAS}

1. BBCMundo. 2008. El dilema de las aguas residuales. Nota de BBCMundo.com [sitio en Internet]. [citado 19 Ago 2008]. Disponible en: http://news.bbc.co.uk/hi/spanish/science/newsid 7567000/7567969.stm

2. UNESCO. Agua para todos, agua para la vida. Resumen. Informe de las Naciones Unidas para el Desarrollo de los Recursos Hídricos en el Mundo. París: UNESCOWWAP; 2003.

3. UNESCO. El agua una responsabilidad compartida. 2do. Informe de las Naciones Unidas sobre el Desarrollo de los Recursos Hídricos en el Mundo. Resumen Ejecutivo. París: UNESCO-WWAP; 2006.

4. World Health Organization. Guidelines for the safe use of wastewater, excreta and greywater. Vol 1. Policy and regulatory aspects. Ginebra: WHO; 2006a.

http://scielo.sld.cu 
5. World Health Organization. Guidelines for the safe use of wastewater, excreta and greywater. Vol 2. Wastewater use in agriculture. WHO, Ginebra: WHO; 2006b.

6. Environment Agency. The microbiology of sewage sludge (2003)-Part 1. An overview of the treatment and use in agriculture of sewage sludge in relation to its impact on the environment and public health. Her Majesty Stationary Office. London, UK: Environment Agency; 2003.

7. Cifuentes E, Blumenthal U, Ruiz-Palacios G, Bennett S, Peasy A. 1994. Escenario epidemiológico del uso agrícola del agua residual: el valle del Mezquital, México. Salud Pública Mex. 1994;36:3-9.

8. Blumental U, Peasey A. Critical Review of epidemiological evidence of the health effects of wastewater and excreta use in agriculture. World Health Organization. London, UK: School of Hygiene and Tropical Medicine; 2002.

9. Blumental U, Peasey A, Ruiz-Palacios G, Duncan Mara D. Guidelines for the microbiological quality of treated wastewater used in agriculture: recommendations for revising WHO Guidelines. Bull WHO. 2000;78:1104-16.

10. Srikanth $R$, Naik D. Health effects of wastewater reuse for agriculture in the suburbs of Asmara City, Eritrea. Inter J Occ Environ Health. 2004;10:284-88.

11. Rai PK, Tripathi BD. Microbial contamination in vegetables due to irrigation with partially treated municipal wastewater in a tropical city. Inter ] Occ Environ Health Res. 2007;17:389-95.

12. Ayres RM, Mara DD. Analysis of waste water for use in agriculture: A laboratory manual of parasitological and bacteriological techniques. Ginebra: WHO; 1996.

13. American Public Health. Standards Methods for the examination of water and wastewaters. Washington: APHA, AWWA; 2005.

Recibido: 30 de abril de 2010.

Aprobado: 12 de mayo de 2010.

María Isabel González González. Instituto Nacional de Higiene, Epidemiología y Microbiología (INHEM). Infanta 1158 e/ Clavel y Llinás. La Habana. Cuba. Tel. (537) 870-5531-34 ext. 143, Fax (53-7) 863-63-20. Correo electrónico: isa@inhem.sld.cu; mariaisa@infomed.sld.cu 